\title{
Isolated Gait Ataxia as a Sole Manifestation of Right Lateral Medullary Infarct following Ipsilateral Trigeminal Herpes Zoster
}

\author{
Seung-Jae Lee Dong-Geun Lee \\ Department of Neurology, Sejong General Hospital, Bucheon, South Korea
}

\section{Key Words}

Herpes zoster $\cdot$ Stroke $\cdot$ Lateral medullary infarct $\cdot$ Vasculopathy

\begin{abstract}
Background: Cerebral infarct related to varicella-zoster virus (VZV) has been reported in the literature. In addition, lateral medullary infarct (LMI) can be manifested rarely as isolated gait ataxia without other characteristic symptoms. Case Report: A 70-year-old female was admitted to our hospital because of herpes zoster on the right trigeminal nerve distribution. On the 15th hospital day, she developed sudden vertigo and fine left-beating nystagmus with axial lateropulsion to the right side, without the other usual signs of LMI (Horner syndrome, dysarthria, swallowing difficulty or hemibody sensory change). Brain MRI showed a small infarct in the far dorsolateral portion of the right rostral medulla and pontomedullary junction. Cerebrospinal fluid study showed a positive VZV IgG antibody. Conclusions: We reported a case of isolated gait ataxia as the sole manifestation of right LMI following herpes zoster on the right trigeminal distribution. A high index of clinical suspicion for concomitant central nervous system complication is necessary in patients with herpes zoster, although the concomitant neurologic symptom may be mild or less lateralized.

(c) 2014 S. Karger AG, Basel
\end{abstract}

\section{Introduction}

Herpes zoster is a common sporadic disease that results from the reactivation of latent varicella-zoster virus (VZV) from dorsal root ganglia [1]. On rare occasions, it can lead to a cerebral infarct by causing cerebral angiitis. There are only a few reports of isolated axial lateropulsion as the sole presentation of lateral medullary infarct (LMI) in the literature [2- 
Lee and Lee: Isolated Gait Ataxia as a Sole Manifestation of Right Lateral Medullary Infarct following Ipsilateral Trigeminal Herpes Zoster

4]. In our report, we present a case of right LMI with isolated lateropulsion following ipsilateral trigeminal herpes zoster.

\section{Case Report}

A 70-year-old, previously healthy female developed excruciating pain on the right cheek and chin. After 3 days, she noticed redness and vesicles on the same area with aggravated pain unresponsive to over-the-counter pain killers. She was admitted to the Neurology Department of our hospital the following day. She had no history of medication, diabetes or chronic hypertension, or alcohol and cigarette use. Physical examination at admission showed paresthesia, allodynia and painful vesicles on the right trigeminal distribution (V2 and V3). Her breathing sound was clear, and the heart beat was regular without murmur. Her blood pressure was 113/73 mm Hg, her pulse rate was 73 beats/min, and her temperature was $36.7^{\circ} \mathrm{C}$. Chest X-ray, electrocardiogram and laboratory findings including a complete blood count and chemistry (renal/hepatic function test, electrolyte, uric acid and lipid profile) were normal. She was treated with intravenous acyclovir ( $15 \mathrm{mg} / \mathrm{kg} /$ day) for 7 days. Pain was controlled by various analgesics (acetaminophen 975-1,950 mg/day, tramadol $112.5-225 \mathrm{mg} /$ day and pregabalin $75-300 \mathrm{mg} /$ day).

While the painful vesicles were resolving, vertigo and gait disturbance with nausea developed 15 days after hospitalization. The patient was able to walk but tended to be lateropulsive to the right side. On neurologic examination, she was alert and oriented. Fine left-beating nystagmus was observed on the left gaze with no limitation of ocular movement and skew deviation. She exhibited no other focal neurologic signs including the usual signs of LMI (Horner syndrome, swallowing or articulation difficulty and hemibody sensory change). At first, we made a presumptive diagnosis of pregabalin-related dizziness or concomitant vestibular neuritis because she showed no focal signs other than lateropulsive gait and nystagmus. The dose of pregabalin was reduced from 300 to $75 \mathrm{mg} /$ day, and a vestibular suppressant (diazepam $2 \mathrm{mg}$ /day) was prescribed. Dizziness was moderately relieved, but mild dizziness persisted thereafter.

Sixteen days later, painful vesicles developed again on the right chin, with high fever $\left(>38^{\circ} \mathrm{C}\right)$ and a headache. Brain computed tomography showed no abnormal findings. Lumbar puncture revealed an opening pressure of $15 \mathrm{~cm}$ of water, 24 red blood cells, 13 white blood cells (lymphocytes $57 \%$, neutrophils $23 \%$, eosinophils $2 \%$ and other $18 \%$ ), glucose $79 \mathrm{mg} / \mathrm{dl}$ (blood glucose $111 \mathrm{mg} / \mathrm{dl}$ ) and protein $37.4 \mathrm{mg} / \mathrm{dl}$. A polymerase chain reaction (PCR) for VZV in cerebrospinal fluid (CSF) was negative. However, VZV IgG antibody was positive in CSF (3.3; normal value $<0.8$ ), and the antibody index of VZV IgG antibody in CSF - the ratio of CSF/serum VZV IgG antibody compared with the CSF/serum total IgG was 63.6 (normal value $<2$ ), which is indicative of intrathecal synthesis. The patient was further treated with a high dose of intravenous acyclovir (30 mg/kg/day) and an oral steroid (prednisolone $1 \mathrm{mg} / \mathrm{kg} /$ day) for 10 days.

Eight days later, brain MRI was performed, which showed a small infarct in the far posterolateral portion of the right rostral medulla and pontomedullary junction (fig. 1). However, there was no abnormal vessel change on brain MR angiography. One month later, the patient recovered with no disability (modified Rankin Scale $=1$ ) but complained of mild transient dizziness upon positional change. 
Lee and Lee: Isolated Gait Ataxia as a Sole Manifestation of Right Lateral Medullary Infarct following Ipsilateral Trigeminal Herpes Zoster

\section{Discussion}

We assumed that stroke occurrence in our patient was related to herpes zoster for several reasons: first, the development of stroke was temporally related to the occurrence of herpes zoster; second, stroke occurred in the brainstem region ipsilateral to the infected side of the trigeminal nerve distribution. In addition, the results of the CSF study suggested a VZV infection of the central nervous system (CNS).

The CSF PCR was negative for VZV in our patient. However, it is well known that the negative PCR result does not exclude the diagnosis of VZV vasculopathy because of its low sensitivity (approx. 30\%) [5]. Instead, VZV IgG antibody was detected in the CSF of our patient. Moreover, the CSF/serum ratio of VZV IgG antibody was significantly increased compared with the CSF/serum ratio of total IgG, suggesting intrathecal synthesis of VZV IgG antibody. Our findings are in agreement with previous reports, which also show the greater diagnostic value of detecting VZV IgG antibody in CSF than that of detecting VZV DNA [5, 6].

Many reports have shown that herpes zoster can be complicated by cerebral angiitis and lead to a cerebral infarct [7-10]. Reactivated VZV might migrate transaxonally or spread hematogenously to intracranial cerebral arteries, resulting in small- or large-vessel vasculopathy $[7,10]$. In our case, the infarct area was anatomically closely related to the ipsilateral spinal trigeminal tract and nuclei (fig. 1c). Therefore, VZV might migrate to CNS transaxonally and then infect small vessels (probably small branches of vertebral or posterior inferior cerebellar artery) supplying the ipsilateral dorsolateral portion of the medulla.

This case is unique in that zoster-related vasculopathy involved only a small area of the ipsilateral dorsolateral portion of the medulla, without evidence of vessel change on MR angiography. To the best of our knowledge, there has been no report of LMI related to herpes zoster in the literature. In addition, our patient had no usual signs of LMI including Horner syndrome, swallowing difficulty, dysarthria or sensory change, which would have prompted an early decision of brain imaging study. Gait ataxia alone was the sole presenting sign of LMI in our patient. This finding initially led to a misdiagnosis of drug-related dizziness or concomitant vestibular neuritis. Therefore, it is recommended that this rare clinical entity of LMI should be included in the differential diagnosis of an isolated vertigo because it mimics peripheral vestibular disorder.

There are a few case reports of isolated gait ataxia as a presentation of LMI [2-4]. Similar to the current case, most of these earlier cases resulted from a small infarct involving the far dorsolateral portion of the rostral medulla (vestibular nuclei and inferior cerebellar peduncle containing the olivocerebellar climbing fiber) [2, 3]. Only one case was presumably related to the inferior olive lesion [4].

In conclusion, we reported a case of LMI related to herpes zoster, presenting only axial lateropulsion with fine nystagmus. A high index of clinical suspicion for concomitant CNS complication is necessary in patients with herpes zoster, although the concomitant neurologic symptom may be mild or less lateralized.

\section{References}

1 Whitley RJ: Varicella-zoster virus infections; in Longo DL, Fauci AS, Kasper DL, Hauser SL, Jameson J, Loscalzo J (eds): Harrison's Principles of Internal Medicine, ed 18. New York, McGraw-Hill, 2012.

2 Kim JS: Vertigo and gait ataxia without usual signs of lateral medullary infarction: a clinical variant related to rostral-dorsolateral lesions. Cerebrovasc Dis 2000;10:471-474.

3 Lee H, Sohn CH: Axial lateropulsion as a sole manifestation of lateralmedullary infarction: a clinical variant related to rostral-dorsolateral lesion. Neurol Res 2002;24:773-774. 
Lee and Lee: Isolated Gait Ataxia as a Sole Manifestation of Right Lateral Medullary Infarct following Ipsilateral Trigeminal Herpes Zoster

4 Kishi M, Sakakibara R, Nomura T, Yoshida T, Yamamoto M, Kataoka M, Ogawa E, Tateno F: Lateral medullary infarction presenting as isolated vertigo and unilateral loss of visual suppression. Neurol Sci 2012;33:129132.

-5 Nagel MA, Cohrs RJ, Mahalingam R, Wellish MC, Forghani B, Schiller A, Safdieh JE, Kamenkovich E, Ostrow LW, Levy M, Greenberg B, Russman AN, Katzan I, Gardner CJ, Häusler M, Nau R, Saraya T, Wada H, Goto H, de Martino M, Ueno M, Brown WD, Terborg C, Gilden DH: The varicella zoster virus vasculopathies: clinical, CSF, imaging, and virologic features. Neurology 2008;70:853-860.

-6 Nagel MA, Forghani B, Mahalingam R, Wellish MC, Cohrs RJ, Russman AN, Katzan I, Lin R, Gardner CJ, Gilden DH: The value of detecting anti-VZV IgG antibody in CSF to diagnose VZV vasculopathy. Neurology 2007;68:1069-1073.

7 Amlie-Lefond C, Kleinschmidt-DeMasters BK, Mahalingam R, Davis LE, Gilden DH: The vasculopathy of varicella-zoster virus encephalitis. Ann Neurol 1995;37:784-790.

8 Ueno M, Oka A, Koeda T, Okamoto R, Takeshita K: Unilateralocclusion of the middle cerebral artery after varicella-zoster virus infection. Brain Dev 2002;24:106-108.

-9 Ortiz GA, Koch S, Forteza A, Romano J: Ramsay hunt syndrome followed by multifocal vasculopathy and posterior circulation strokes. Neurology 2008;70:1049-1051.

10 Gilden DH, Lipton HL, Wolf JS, Akenbrandt W, Smith JE, Mahalingam R, Forghani B: Two patients with unusual forms of varicella-zoster virus vasculopathy. N Engl J Med 2002;347:1500-1503.

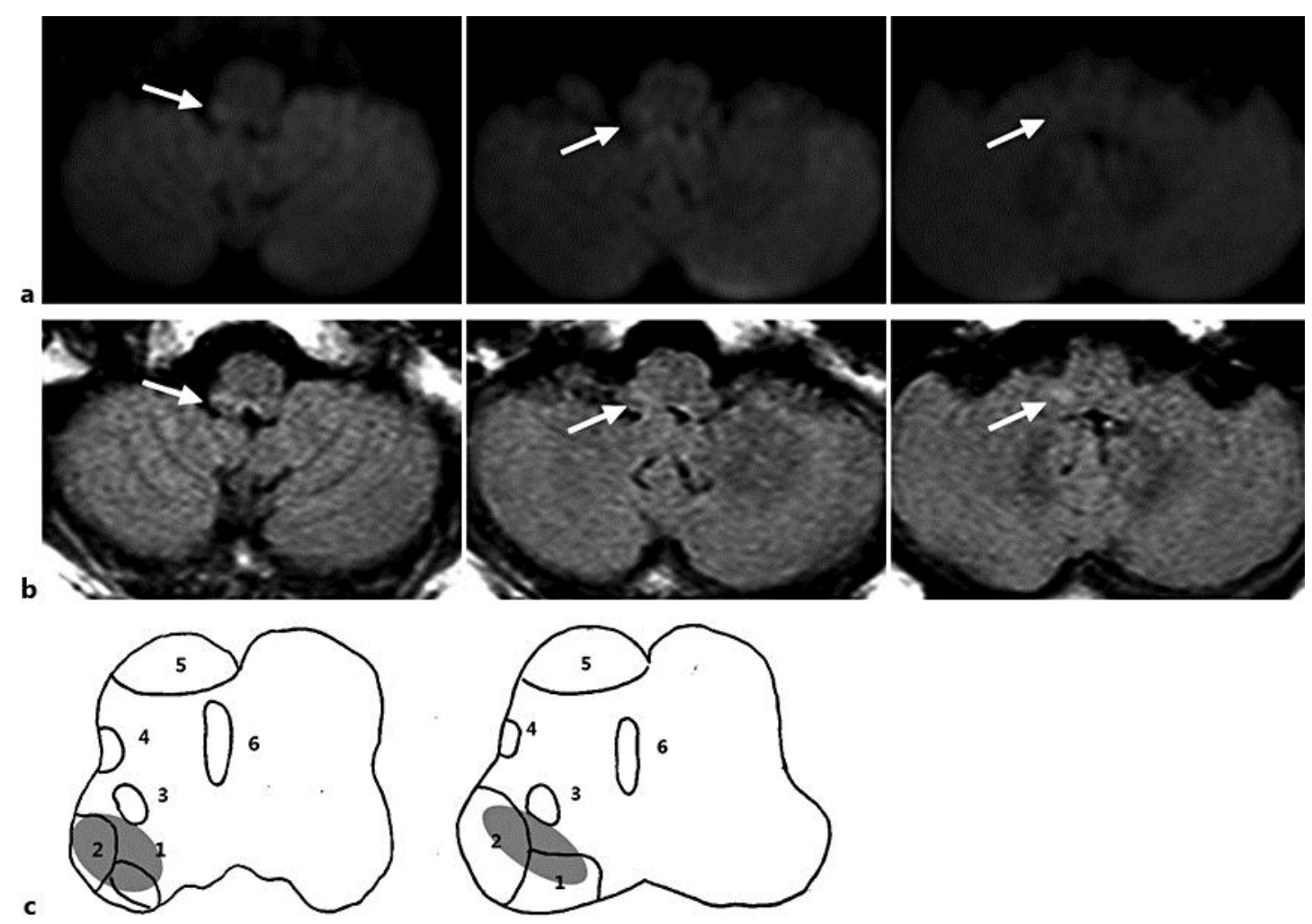

Fig. 1. Brain MRI of the patient. Axial slices of diffusion-weighted (a) and fluid-attenuated inversion recovery (b) imaging showed a small infarct in the far dorsolateral portion of the rostal medulla and pontomedullary junction, involving the inferior cerebellar peduncle and vestibular nuclei (arrows). c Schematical cross-sectional anatomy of the rostral medulla (left) and pontomedullary junction (right). The shaded areas in $\mathbf{c}$ indicate the infarct region. $1=$ Vestibular nuclei, $2=$ inferior cerebellar peduncle, $3=$ spinal trigeminal tract and nucleus, $4=$ spinothalamic tract, $5=$ pyramid, $6=$ medial lemniscus. 